\title{
Entrevista
}

\section{OSTI-DOE, la fuente de información sobre energía. Entrevista a Karen Spence}

\author{
Por Fernanda Peset y Tomàs Baiget
}

\begin{abstract}
Resumen: Entrevista a Karen Spence, subdirectora de Sistemas de Información de la Office of Scientific and Technical Information (OSTI), Department of Energy, de los Estados Unidos, que sirve para introducirnos en las importantes bases de datos, repositorios y proyectos de cooperación en información científica y técnica (especialmente sobre energía y medio ambiente) que realiza dicha Oficina.

Palabras clave: Información científica y técnica, Energía, Medio ambiente, Bases de datos, Web, Repositorio, Cooperación, Department of Energy, DOE, Office of Scientific and Technical Information, OSTI.

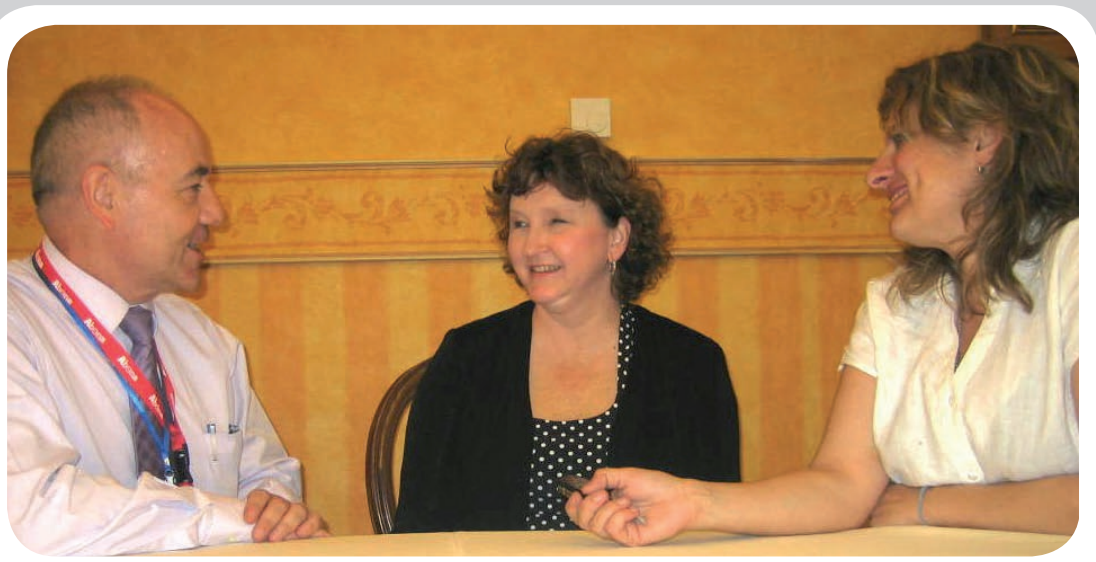

Karen Spence (centro) entrevistada por Tomàs Baiget y Fernanda Peset

\section{Title: OSTI-DOE, the source of energy information. Interview with Karen Spence}

Abstract: An interview with Karen Spence, assistant director of Information Systems, Office of Scientific and Technical Information (OSTI), United States Department of Energy, describes the major OSTI databases, repositories and scientific and technical information co-operation projects (especially on energy and environment).
\end{abstract}

Keywords: Scientific and technical information, Energy, Environment, Databases, Web, Repository, Co-operation, Department of Energy, DOE, Office of Scientific and Technical Information, OSTI.

Peset, Fernanda; Baiget, Tomàs. "OSTI-DOE, la fuente de información sobre energía. Entrevista a Karen Spence”. El profesional de la información, 2009, septiembre-octubre, v. 18, n. 5, pp. 563-566.

DOI: $10.3145 /$ epi.2009.sep.12

LA OFFICE OF SCIENTIFIC AND TECHNICAL INFORMATION (OSTI), del US Department of Energy, es una de las instituciones clásicas y pioneras de la documentación (en 2007 celebró su $6^{\circ}$ aniversario), que por los años 50 y 60 desarrolló teorías y prácticas que sirvieron de modelo a las primeras generaciones de profesionales de bibliotecas especializadas que empezaban a distinguirse de los bibliotecarios, asentando los fundamentos de la Documentación o Information science.

Más tarde, en los 80 y 90 fue famosa por su base de datos sobre energía, aunque en muchos países no pudimos usarla debido a determinados pactos de colaboración con agencias corresponsales (en España la Junta de Energía Nuclear, hoy Centro de Investigaciones Energé- ticas Medioambientales y Tecnológicas, Ciemat), que la consideraron como propia, no abierta al público - cosas típicas de las políticas de otras épocas, que también sucedió durante unos años con la base de

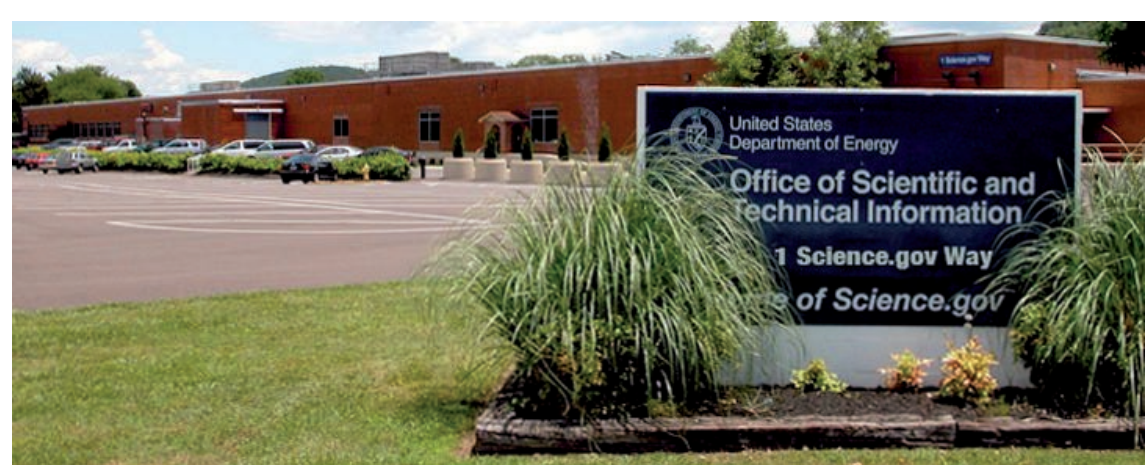

Instalaciones de la OSTI en Oak Ridge, Tennessee 
datos Agris, de la $F A O$, cuyo representante en España era el INIA-.

Hemos aprovechado la visita a España de Karen Spence, subdirectora de Sistemas de Información, de la OSTI, para entrevistarla y además para ayudar a difundir unas bases de datos importantes y poco conocidas:

\section{¿Qué es y qué hace la OSTI?}

- Es una agencia del Departamento de Energía del Gobierno de los EUA que siempre ha tenido una gran vocación en el tema de la documentación y de las bases de datos. Es claro que la Energía (y todos sus temas relacionados) es una materia muy importante, pero además tradicionalmente la $O S T I$ ha estado bastante por delante de otras agencias. Tenemos un lema famoso: "El saber se contagia: nuestra misión es que todo el mundo lo pille". Si el intercambio de conocimientos se acelera, el progreso científico también se acelera y las décadas se comprimen a años, los años a meses, y los meses a días...

Hemos visto varias referencias al "bien público" en documentos de la OSTI. ¿Qué importancia dáis a este concepto?

- Como es sabido, un bien público es uno que no disminuye en valor con el uso, y (casi) no tiene coste marginal para los usuarios después de la primera utilización.

Un bien público científico es un caso especial, pues su valor aumen$t a$ con el uso. Por consiguiente, para aprovechar al máximo el uso de la información generada por la investigación, es imprescindible que sea un bien público.

En la OSTI propugnáis el open access, pero luego muchas veces son los técnicos los que retienen la información.

- Sí, y esto se acepta para períodos de tiempo que permitan a los investigadores explorar suficiente- mente las consecuencias y posibilidades de sus descubrimientos. Lo mismo que ocurre con las pequeñas empresas, los grupos pequeños de investigación pueden ser más lentos para seguir y evaluar las consecuencias inmediatas de su investigación, por lo que dicha auto-protección es bastante aceptable.

De todas maneras, como dice Ángel Borrego, de la Universidad de Barcelona, se produce el síndrome Jekill-Hyde: los científicos se comportan de forma diferente según sean lectores o autores.

\section{¿Planes para el futuro?}

- Técnicamente ahora mismo la OSTI tiene un peso muy importante entre las demás agencias investigadoras de los EUA sobre todo por nuestra aportación a las plataformas de difusión, pero estamos siguiendo de cerca los pasos de otros, como por ejemplo los $\mathrm{Na}$ tional Institutes of Health (NIH), y su mandato a los investigadores que subvenciona para que hagan públicos sus resultados. Nos parece muy buena la sugerencia de que los autores firmen su cesión del copyright en estos términos: "La revista reconoce que el autor retiene el derecho a proporcionar una copia del manuscrito final a los NIH cuando la revista acepte publicar el artículo, o posteriormente, para el archivo público en PubMed Central tan pronto como sea posible después de la publicación en la revista". ca?

¿Se notará la crisis económi-

- Esperamos que no demasiado. El despliegue tecnológico de la OSTI para el acceso a la información siempre se ha desarrollado con una utilización lo más juiciosa posible de unos fondos más bien limitados. Su presupuesto anual es de 9 millones US\$, que representa sólo el $0,1 \%$ del presupuesto para I+D del DOE.

\section{Productos y servicios de información}

\section{DOE Information bridge}

DOE Information bridge (1991) ofrece libre acceso a 170.000 informes en texto completo, desde 1991, producidos por el DOE y sus contratistas, sobre física, química, materiales, biología, ciencias ambientales, energía, ingeniería, informática, ciencias de la información y energías renovables. A la derecha de la captura de pantalla se distingue un apartado en el que se puede opinar y discutir sobre cada informe.

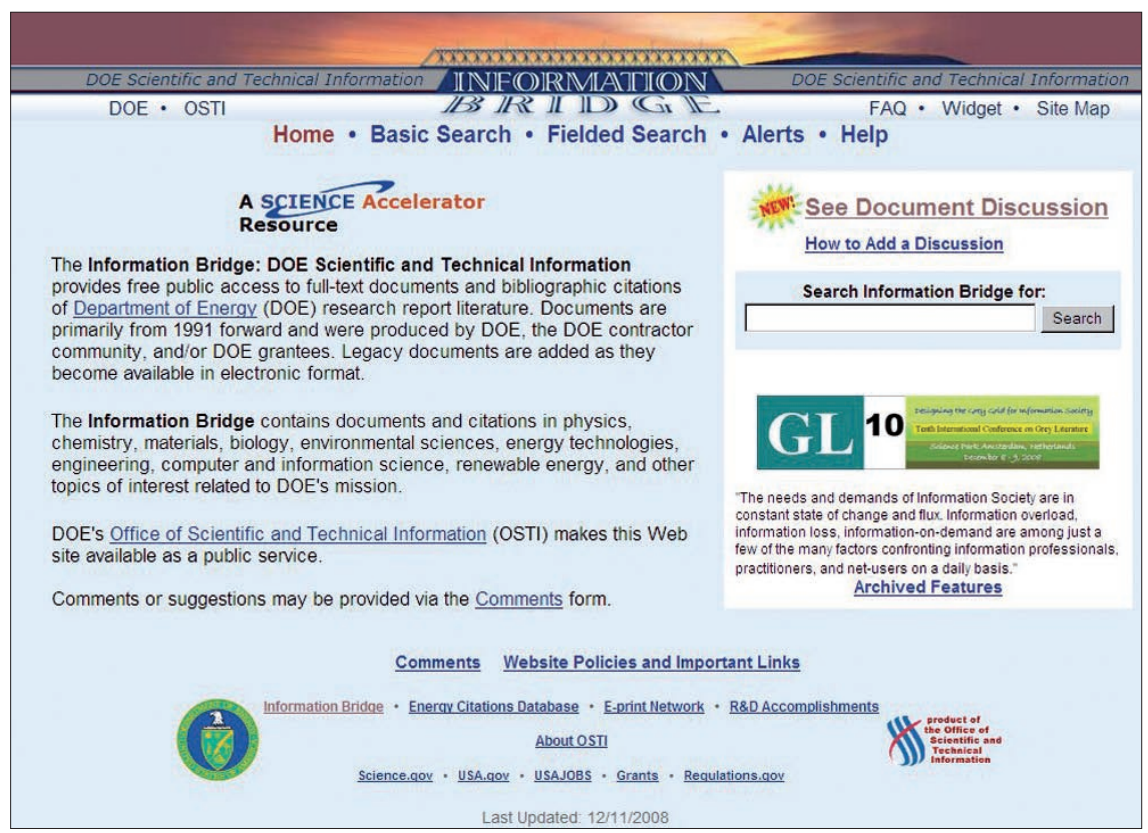

http://www.osti.gov/bridge 


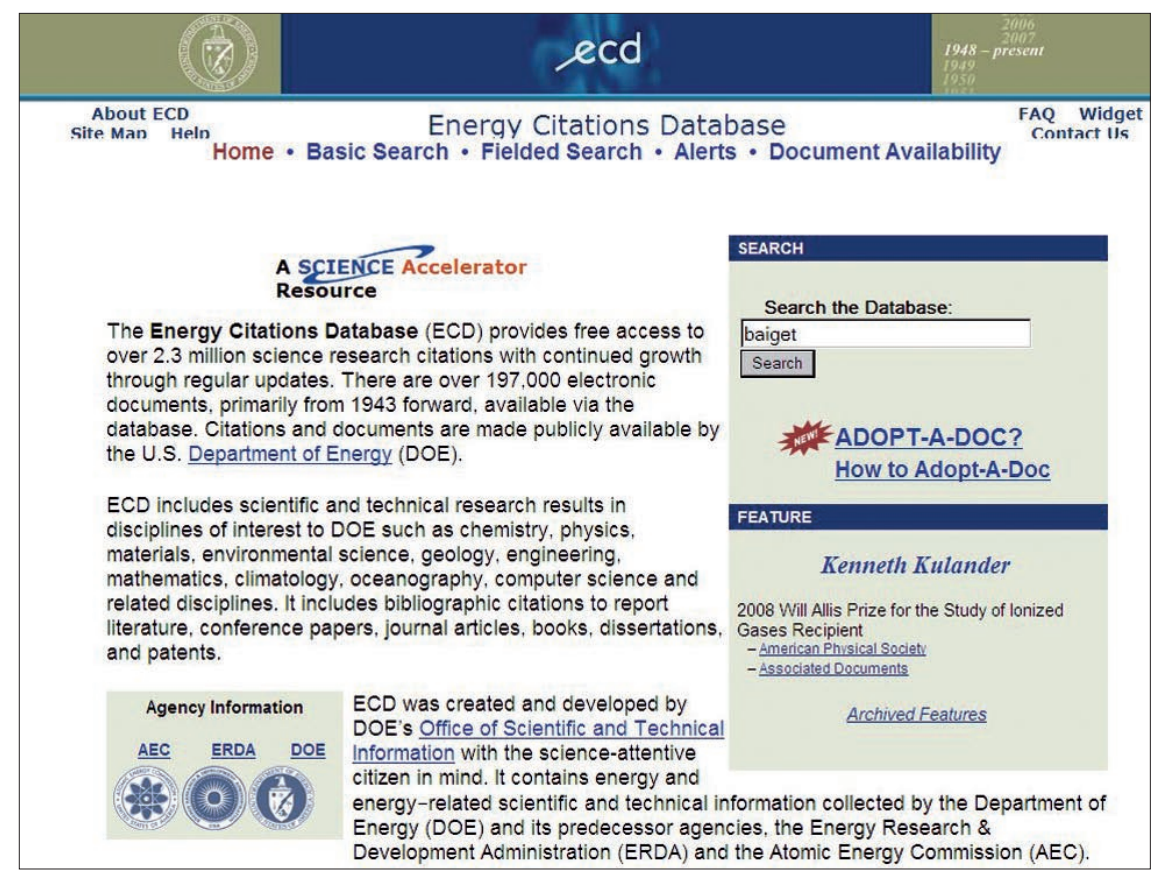

http://www.osti.gov/energycitations ses de datos (sobre las que se hacen búsquedas federadas o simultáneas), y provee enlaces a 3.000 sociedades profesionales.

OSTI quiere hacer evolucionar $E P N$ de manera que de ser una fuente básica de información pase a ser un servicio de apoyo al investigador en el sentido más amplio, en todas sus necesidades de información profesional.

Por ejemplo podría utilizarse para:

- dar soporte a actividades de información paralelas como la creación, almacenamiento, conservación y archivo de documentos;

- construcción automática de

\section{Energy citations database}

Energy Citations Database (1943-) contiene 2,3 millones de referencias bibliográficas de informes, ponencias, artículos, libros, tesis, y patentes de interés para el DOE, y sus agencias predecesoras [la Energy Research \& Development Administration (ERDA) y la Atomic Energy Commission (AEC)].

\section{Colaboración entre agencias del Gobierno}

\section{Science.gov}

Es una coalición voluntaria de 12 agencias federales, aunque entre ellas OSTI ocupa un lugar preeminente.

Ofrece acceso a 40 bases de datos y 1.950 sitios web científicos, con un total de 200 millones de páginas.

Realmente Science.gov es una mina de información muy poco conocida en Europa, por lo que recomendamos a nuestros lectores que la tengan presente cuando tengan que buscar información científicotécnica, especialmente si tiene que estar relacionada con las administraciones públicas.

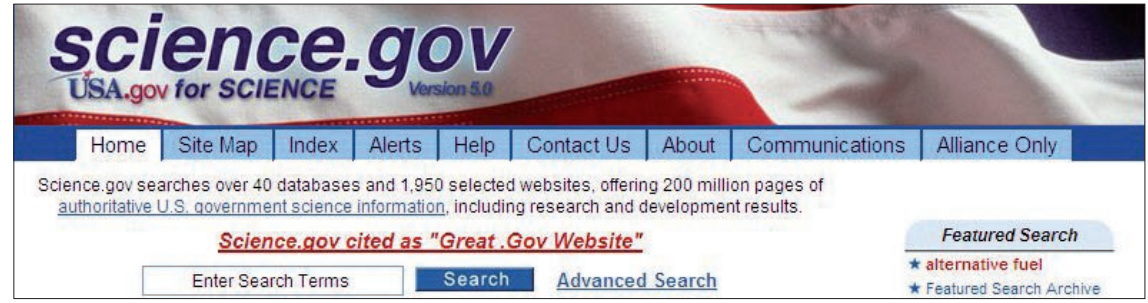

http://www.science.gov/

\section{Colaboración internacional}

\section{E-prints Network}

Iniciado en enero de 2000, $E$ prints Network (EPN) se ha convertido en uno de los principales productos de OSTI.

Proporciona acceso a 26.000 sitios web, con 1 millón de e-prints a texto completo, y 3 millones de documentos adicionales de 52 ba- estrategias de búsqueda basadas en búsquedas previas que hayan dado buenos resultados;

- uso de software para gestión del conocimiento sobre la colección de documentos;

- localización de expertos;

- búsqueda de puestos de trabajo, becas, subvenciones;

y así sucesivamente.

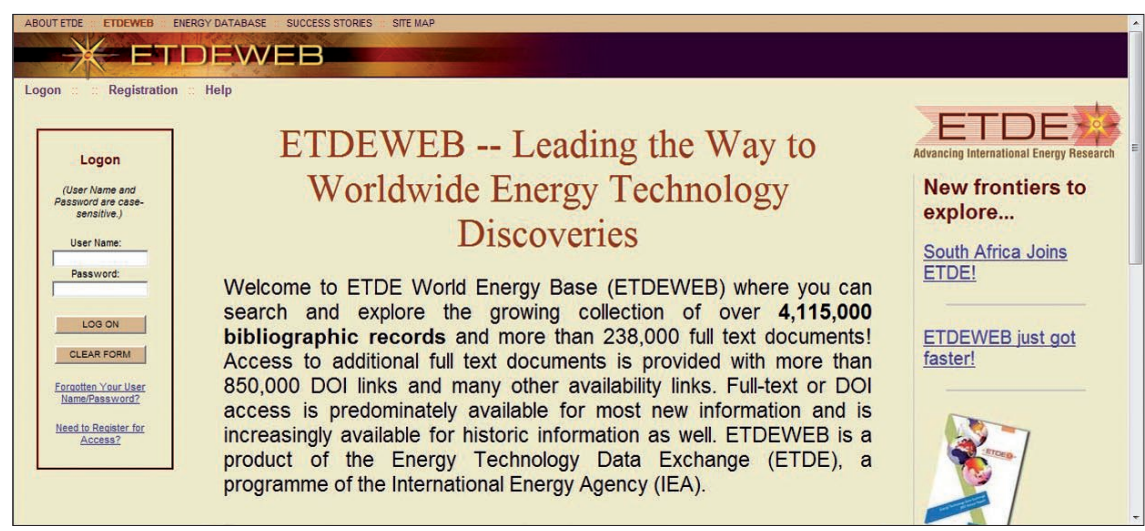

http://www.etde.org/ 


\section{ETDE Web}

El proyecto Energy Technology Data Exchange es parte de la International Energy Agency (IEA), y la OSTI constituye su motor gestionándolo.

Con 4,2 millones de referencias (1974-), es la base de datos más grande del mundo sobre energía. "EtdeWeb es claramente el sistema de información de referencia sobre energía, medio ambiente y temas relacionados", proclamaba la $\mathrm{Co}$ misión Europea en mayo de 2005.

EtdeWeb tiene integrada la antigua base de datos DOE Energy (distribuida por Dialog con el $n^{\circ} 103$ ).

De 238.000 de las citas se puede obtener directamente el correspondiente texto completo en pdf. Además se incluyen 850.000 DOIs que permiten también localizar el pdf en editoriales de pago.

El acceso está restringido a los países miembros de la IEA colaboradores de ETDE. España es miembro fundador y los españoles podemos usar este servicio después de rellenar un formulario online, y recibir la aprobación del Ciemat. En Latinoamérica los miembros de ETDE son Brasil y México, aunque otros países también pueden acceder a la base de datos.

\section{Global Science Gateway}

Como se recordará, la declaración de intenciones para crear este
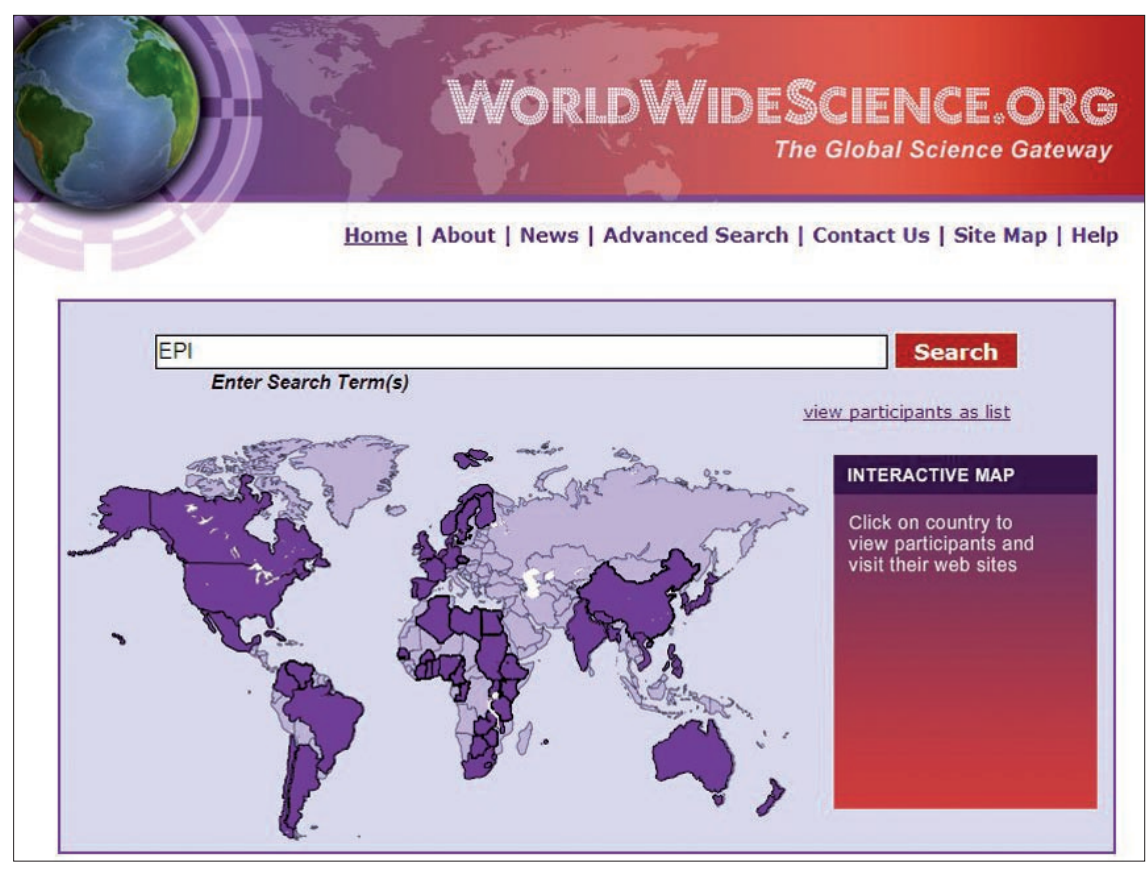

http://worldwidescience.org/

portal mundial de la ciencia fue firmada el 21 de enero de 2007 por Raymond Orbach, subsecretario de Ciencia del $D O E$, y Lynne Brindley, directora ejecutiva de la British Library.

La OSTI se encarga de su operación.

Actualmente el servicio se llama World Wide Science, y con la adición de Corea (junio 2008) y China (octubre 2008), da acceso a 32 repositorios (entre ellos E-LIS) de 44 países, que totalizan $200 \mathrm{mi}$ llones de documentos.

Karen Spence, Assistant Director for Information Systems, Office of Scientific and Technical Information, US Department of Energy.

Tel.: +1-865 57402 95; fax: 8655769357

spencek@osti.gov

http://www.osti.gov

http://www.science.gov/workshop/ spencek.html

Fernanda Peset, Univ. Politécnica de Valencia, Depto. de Comunicación Audiovisual, Documentación e Historia del Arte (Dcadha), Camino de Vera, s/n. 46022 Valencia. mpesetm@upv.es

Tomàs Baiget, EPI SCP, Apartado 32280, 08080 Barcelona.

baiget@sarenet.es

\section{Suscripciones}

Renovar (o comenzar) la suscripción a "El profesional de la información" es ágil y sencillo.

Usted puede gestionar online su suscripción conectándose a esta web: http://www.elprofesionaldelainformacion.com/suscripciones.html

Si lo desea puede comunicar con nosotros dirigiéndose a: suscripciones@elprofesionaldelainformacion.com

o al teléfono: +34-609352954 